\section{ScienceDirect}

Materials Today: Proceedings 4 (2017) 5779-5784 materialstoday:
PROCEEDINGS

www.materialstoday.com/proceedings

DAS 2016

\title{
Morphological and structural evaluation of trabecular bone in early and late stage of osteoarthritis
}

\author{
Francesca Cosmi ${ }^{\mathrm{a} *}$, Magdalena Tomanik ${ }^{\mathrm{b}}$ \\ ${ }^{a}$ University of Trieste, Department of Engineering and Architecture, Via A. Valerio 10, 34127, Trieste, Italy \\ ${ }^{b}$ Wroctaw University of Science and Technology, Department of Biomedical Engineering, Mechatronics and Theory of Mechanisms, \\ Lukasiewicza 7/9, 50-371 Wrocław, Poland
}

\begin{abstract}
Osteoarthritis (OA) is a degenerative joint disease that occurs mostly in the knee and in the hip. It is expected that more than $20 \%$ of the population over the age of 60 in Europe and in the United States will show signs and symptoms associated with OA, and that one-third of OA patients will experience disability and immobilization, hence the motivation for this research aimed at achieving new insights into the bone structure alterations induced by this disease. In an original approach, the 3D reconstructions obtained by high-resolution X-ray micro-CT were the starting point for: i) a morphological examination and 2) structural 3D analyses by numerical simulation of the bone architectures behavior, thus providing an extended description of the changes in osteoarthritic bone as disease progress. This paper presents the first results obtained by the combination of morphological and structural 3D analyses from on high-resolution micro-CT reconstructions of trabecular bone in early and late stage hip OA.
\end{abstract}

(C) 2017 Elsevier Ltd. All rights reserved.

Selection and/or Peer-review under responsibility of 33rd Danubia Adria Symposium on Advances in Experimental Mechanics.

Keywords: osteoarthritis; cell method; structural paramieters; morphological analisys.

\section{Introduction}

Osteoarthritis (OA) is a degenerative joint disease and occurs mostly in the knee and in the hip. It is expected that in the future more than $20 \%$ of the population over the age of 60 in Europe and in the United States will show

\footnotetext{
* Corresponding author. Tel.: +39-040-5583431; fax: +39-040-558-3812.

E-mail address: cosmi@units.it
} 


\begin{tabular}{|ll}
\hline Nomenclature \\
BS & Bone Surface \\
BV/TV & Bone Volume/Total Volume \\
CM & Cell Method \\
DA & Degree of Anisotropy \\
E & Young modulus \\
OA & Osteoarthritis \\
SMI & Structure Model Index \\
Tb.N & Trabecular Number \\
Tb.Sp & Trabecular Separation \\
VOI & Volume of interest \\
\hline
\end{tabular}

signs and symptoms associated with OA, and that $30 \%$ of OA patients will experience disability and immobilization, hence the motivation for this research aimed at achieving new insights into the trabecular bone modifications induced by this disease.

The affected joints typically undergo characteristic changes involving narrow space between the joints, degeneration of the cartilage and cysts formation in the subchondral bone. The cartilage loss observed during the progression of osteoarthritis is generally used as an adequate parameter for ranking the different stages of the disease. Two important classification criteria [1,2], based on joint surface changes, have been used for over a half century to determine the stage of OA. The severity of the disease is evaluated by describing the increasing area of the destructed cartilage as well as the morphological chances in cartilage tissue, such as the softening and the swelling of the cartilage leading to erosion and finally exposure of subcortical bone. Osteoarthritis begins with an enzymatic breakdown of the cartilage matrix caused by the affected chondrocytes metabolism.

Horizontal and vertical fibrillation of the cartilage occurs in a later stage, when the articular surface becomes eroded and the matrix fragments are released into the joint cavity and induce inflammation. Due to the repairing process, the bone is stimulated to form new tissue. This bone growth affects not only the epiphysis but also the joint surface [3], inducing biological changes within the femoral bone that have an impact on the entire biomechanical conditions of the joint. Even small cartilage losses can cause a ten times increase of the pressure load acting in the epiphysis, prompting the remodeling mechanism of the trabecular bone in order to adjust the geometry of the epiphysis to the new loading conditions and to provide stability in the joint. This remodeling process has a significant impact on both the structural and mechanical trabecular properties.

Although it is generally assumed that damage of the subchondral bone is secondary to cartilage damage, recent studies have shown that bone changes are recognizable also in the early stages of OA disease, and even precede the alteration of the cartilage [4]. These findings seem to be confirmed also by observations in animal studies $[5,6]$.

Currently, OA diagnosis is mostly based on radiological examination. The affected joints typically undergo characteristic changes involving narrow space between the joints, degeneration of the cartilage and cysts formation in the subchondral bone [7,8]. Diagnosis imaging examinations including MRI or CT are more sensitive in early OA but rarely used because of their high cost. Nevertheless, clinical CT has shown a great ability to assess imaging features of OA (Fig.1) and to provide information regarding the disease stage [9].

Enhanced quantitative assessment of bone tissue histomorphology can indeed provide valuable information about the nature of the bone changes in the hip. Moreover, quantification of bone tissue properties allows a better understanding of the disease progression [10], providing additional data which may lead to developing new methodologies for prevention and treatment of OA.

Recent epidemiological studies [11] have suggested that two independent phenomena contribute to the OA degenerative joint disease, indicating that increase in bone mineral density is a risk factor for OA and that the rate of bone loss in people with radiographic diagnosis of OA is altered. While there is a growing interest in improving our understanding of the mechanisms underlying bone alterations as a risk factor for a better diagnosis of hip arthritis, the mechanisms underlying these observations still remains unclear and should be investigated.

This paper presents the first results obtained by a combination of two approaches: morphological and structural 3D analyses, based on high-resolution micro-CT reconstructions of trabecular bone in early and late stage hip OA. 


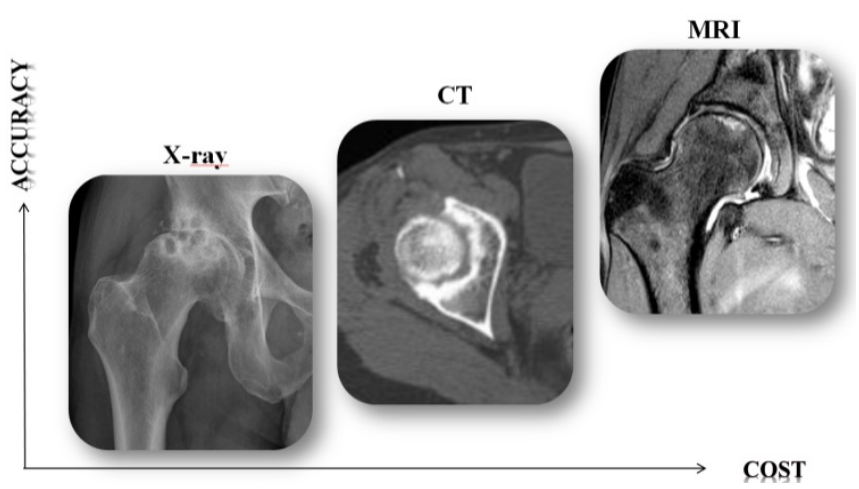

Fig.1. Dependence of the accuracy of the different imaging techniques in comparison to the cost of the procedure

\section{Materials and Methods}

The subjects of this study were 6 proximal femoral epiphyses collected from patients who had undergone total hip arthroplasty for osteoarthritis in University Clinical Hospital in Wrocław, Poland, were divided into two groups respectively representing early $(n=3)$ and late $(n=3)$ stage of hip OA, according to the Outerbridge recognized OA grading system $[1,2]$. Besides the medical diagnoses, the epiphyses were examined to establish the articular cartilage degree of erosion and of fibrosis and to observe changes in the epiphysis geometry.

\subsection{Sample preparation}

From the central part of each epiphysis, a slice of $10 \mathrm{~mm}$ thickness was cut off (Fig.2) using a metallic cutter STRUERS Accutom-5® with diamond blade. Cubic bone specimens with a nominal side of $10 \mathrm{~mm}$ (Fig.2C) were successively cut from the subchondral slice of the femoral head approximately 3-5 mm below the joint surface depending on the femoral head shape, with an axis coincided with the femoral neck shaft direction. The material was stored in $-20^{\circ} \mathrm{C}$ in polyethylene plates until the further testing.
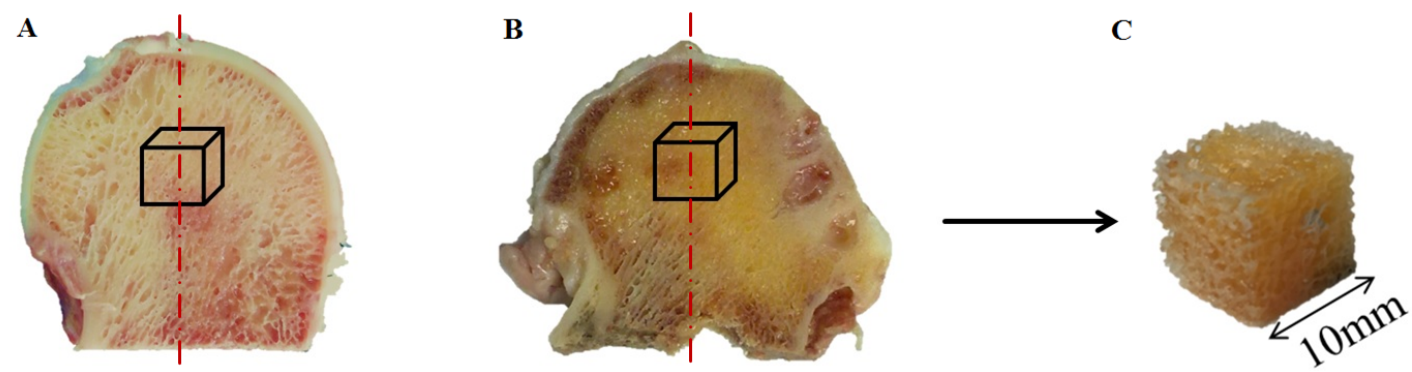

Fig. 2. Images of the femoral heads in (A) early and (B) late stage of OA with (C) cubic trabecular bone specimen selection

\subsection{Micro-CT}

Bone specimens were scanned with high-resolution X-ray micro-CT SkyScan1172, Bruker ${ }^{\circledR}$ (Wrocław, Poland) with a $13.5 \mu \mathrm{m}$ pixel size $(84 \mathrm{kV}, 128 \mu \mathrm{A}, 0.5 \mathrm{~mm}$ Al filter). This nondestructive method allows the reconstruction of the internal trabecular structure of the samples. After segmentation with the Otsu threshold method, the 3D images were scaled down in order to obtain the cubic Volumes Of Interest (VOI), 200 pixel side, suitable for the numerical simulations. The same reconstructed trabecular VOIs were used for the morphological analyses and for the numerical simulations. 


\subsection{Morphological analysis}

Parameters used to characterize the structure morphology of the cancellous bone tissue are standardized and can be divided into three groups related with i) geometry described by areas and volumes of both tissue and bone; ii) structure defined by trabecular thickness, number and shape; iii) anisotropy providing information about the presence/absence of a privileged direction in trabecula arrangement. Using a dedicated software (CTAnn, Bruker) for morphometric analysis, three-dimensional bone architecture parameters, described below, were calculated [12].

\subsection{Cell Method structural analysis}

The Cell Method (CM) is a numerical method based on a direct discrete formulation of equilibrium equations [13]. Due to the direct discrete approach employed, no restriction is imposed by differentiability conditions and the characteristic length of the elementary cell of the discretization can be of the same order of magnitude as the heterogeneities of the structure, so that it is very efficient for the analysis of complex, porous structures.

A Cell Method model, developed in Trieste (Italy) for the simulation of compression tests along the coordinate axes of a trabecular bone volume and described in detail in [14], was applied in this work. The approach has already been successfully applied for the quantitative assessment of the changes in the meso-scale mechanical properties of bone explants due to long-term exposure to simulated weightlessness [15-17].

In this work, an elastic modulus of $1000 \mathrm{MPa}$ and a Poisson's coefficient of 0.3 were assumed for the bone/solid phase material for both early and late stage of OA, and the elastic modulus in each one of the 812,905 tetrahedral cells of the mesh was determined by a scaling procedure (Fig.3). Results of the simulations were the elastic moduli along the VOI coordinate axes.
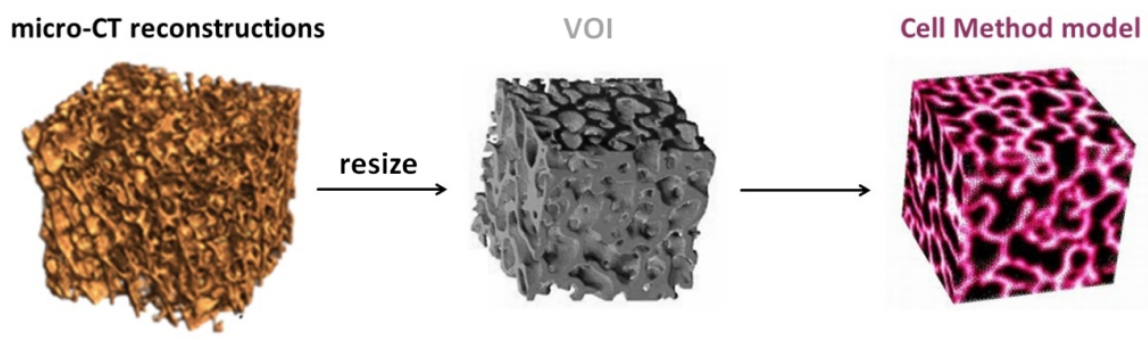

Fig. 3. Conversion of the cubic volumes of interest for the elastic compression test simulations

\section{Results}

The following 3D parameters were calculated in the morphological analyses:

- Bone Volume/Tissue Volume (BV/TV), bone tissue (binarized objects) percentage in the VOI, comparable to volume density in stereological terminology;

- Bone Surface (BS), surface area of all the solid objects within the VOI, measured in 3D based on the marching cubes method;

- Structural Model Index (SMI), structure model index, indicates the relative prevalence of rods and plates in a 3D structure and aims at describing the type of the trabecula building the bone tissue (for a structure built only from plate elements the value is 0 while for a purely rod structure the SMI equals 3);

- Trabecular Number (Tb.N), structure linear density or trabecular number, that is the number of traversals across a trabecular or solid structure per unit length on a random linear path through the VOI

- Trabecular Seperation (Tb.Sp) separation between trabecula's, or thickness of the spaces as defined by banalization within the VOI;

- Degree of Anizotropy (DA_CT) is the degree of anisotropy as determined by the mean intercept length eigenvalues. 
Results of the Cell Method simulations were:

- $\mathrm{E}_{\mathrm{z}}$, the elastic modulus along the neck shaft axis;

- $\mathrm{E}_{\mathrm{xy}}$, the average elastic modulus in the transversal plane.

Additionally, the mean intercept length anisotropy (DA_CT) and the elastic degree of anisotropy (DA_CM) were computed according to Eq. (1)

$$
\mathrm{DA}=1-\left[\frac{\mathrm{X}_{\min }}{\mathrm{X}_{\max }}\right]
$$

where $X_{\min }$ and $X_{\max }$ respectively represent the minimum and the maximum parameter values obtained in the directional mean intercept length analyses and in the $\mathrm{CM}$ simulations. A perfect isotropy yields $\mathrm{DA}=0$, while unidirectionality is indicated by $\mathrm{DA}=1$.

The parameters describing the trabecular structure in the early and in the late stage of OA (average of the 3 samples from each group) are summarized in Tab.1.

Table 1. Morphological and numerical results, average of the 3 samples from each group.

\begin{tabular}{|c|c|c|c|c|c|}
\hline Analysis & Parameter & Unit & Early stage $\mathrm{OA}$ & Late stage OA & $\%$ variation \\
\hline \multicolumn{6}{|l|}{ MORPHOMETRIC } \\
\hline \multirow{6}{*}{ 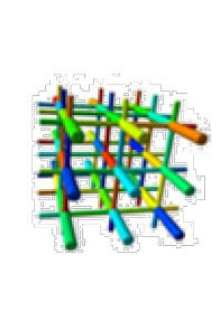 } & $\mathrm{BV} / \mathrm{TV}$ & $\%$ & 21.80 & 24.03 & 10.2 \\
\hline & BS & $\mathrm{cm}^{2}$ & 910 & 1014 & 11.4 \\
\hline & SMI & - & 0.73 & 0.86 & 17.8 \\
\hline & Tb.N & $1 / \mathrm{mm}$ & 0.18 & 0.20 & 11.1 \\
\hline & Tb.Sp & $\mathrm{mm}$ & 4.40 & 3.80 & -13.6 \\
\hline & DA_CT* & - & 0.49 & 0.56 & 14.3 \\
\hline \multicolumn{6}{|l|}{ STRUCTURAL } \\
\hline & $E_{z}$ & $\mathrm{MPa}$ & 124 & 156 & 25.8 \\
\hline & $E_{x y}$ & $\mathrm{MPa}$ & 88 & 105 & 19.3 \\
\hline & DA_CM* & - & 0.29 & 0.33 & 13.8 \\
\hline
\end{tabular}

\section{Conclusion}

Both the morphological and structural evaluation of our trabecular bone sample in early and late stage of osteoarthritis confirmed that changes in the trabecular structure can be evidenced with the progression of OA.

The results obtained from the morphological analyses show an increase in bone volume and in bone surface $>$ $10 \%$, indicating that the structure becomes more packed. Moreover, the results are in good agreement with the expectations of increase in trabecula number and decrease in separation distance between elements. Finally, transformation from plate to rod morphology of the trabeculae was detected, as evidenced by the SMI morphological parameter changes.

The degree of anisotropy values show that directionality is present within the structure of the femoral head. The morphological and the structural DA increase in late stage OA in a similar way, indicating that the trabecular bone remodels itself and changes its structural characteristics as the OA develops. This observation can lead to new insights into the disease progression, since it can be explained by two conceivable, possibly concomitant, scenarios:

- as a mechanism to adjust to changes in the direction of the loading force;

- as a process derived by specific alterations in the trabecular structure organization. 
The morphological results are generally coherent with the changes measured in elastic modulus values, which are in turn related to the yield strength of human trabecular bone [18]. It should be noted, however, that the structural elastic modulus values show larger differences between late stage and early stage samples than the morphological parameters.

Overall, it appears to be confirmed what already pointed out in [16]: an accurate representation of trabecular bone modifications must consider the changes in the mechanical characteristics of the trabecular bone structure, which can only partially be related to the modifications in trabecular morphology.

It is also interesting that the biggest structure elastic modulus changes occur along the femoral neck shaft direction $z$. The OA disease thus appears to affect mostly the properties in the direction that physiologically carries the largest loads, a result that is again coherent with what found in micro-g bone assessment works $[15,16]$. In effect, despite the different mechanisms underlying the bone structural alterations considered, in both cases the effects on bone tissue are ultimately linked to modifications related to the cell response to mechanical stimuli.

In conclusion, while our findings confirm the rationality of the methodological approach adopted, there is a strong motivation for further investigation in order to ratify these results on a larger number of samples.

\section{Acknowledgements}

All the patients gave consent and the study was approved by the bioethics committee of Wrocław Medical University, PL. Funding from UNITS FRA2015 to FC is acknowledged.

\section{References}

[1] DH Collins, The pathology of articular and spinal diseases London:Edward Arnold Co, 1949.

[2] RE. Outerbridge, J Bone Joint Surg 43B (1961): 752-757.

[3] T. Waldron Palaeopathology. Cambridge (2008).

[4] T. Funck-Brentano, M. Cohen-Solal. Curr Opin Rheumatol. 27(4)(2015):420-6.

[5] M. Bellido, Lugo L, et al. Arthritis Res Ther.;12(2010):R152.

[6] E. Calvo, S. Castañeda, et al. Osteoarthritis Cartilage.15(2007):69-77.

[7] T. Conrozier et al., Br J Rheumatol., 37 (1998): 961-968.

[8] T Conrozier. et al., Osteoarthr. Cartil., 9 (2001): 281-286.

[9] T D Turmezei. et. al., Osteoarthr. Cartil 22 (2014): 1360-1366.

[10] M. Tomanik et.al. J Mech Behav Biomed Mater 64 (2016): 86-93.

[11] Hardcastle SA et al. BoneKEy Reports (2015): online.

[12] DW. Depster et al., J Bone Miner Res,. 28 (2013): 1, 2-17.

[13] Tonti E. CMES: Computer Modeling in Engineering \& Sciences. 2(2) (2001): 237-258.

[14] F. Cosmi. D. Dreossi, Meccanica, 42 (2007): 85-93.

[15] F. Cosmi et al., J Mech Behav Biomed Mater, 2 (2009): 164-172.

[16] F. Cosmi et al., J Mech Behav Biomed Mater, 44 (2015): 61-70.

[17] F. Cosmi, M. Tomanik Materials Today- Proceedings 3(4) (2016): 947-952 .

[18] J. Wang, et.al., Bone 72 (2015): 71-80. 